\title{
BMJ Open Vitamin D supplementation prior to in vitro fertilisation in women with polycystic ovary syndrome: a protocol of a multicentre randomised, double-blind, placebo-controlled clinical trial
}

Kai-Lun Hu, ${ }^{1}$ Kwanghann Gan, ${ }^{1}$ Rui Wang (1) , ${ }^{2}$ Wentao $\mathrm{Li}^{2}$ Qiongfang Wu, ${ }^{3}$ Beihong Zheng, ${ }^{4}$ Libo Zou, ${ }^{5}$ Su Zhang, ${ }^{6}$ Yifeng Liu, ${ }^{1}$ Yiqing Wu, ${ }^{1}$ Ruixue Chen, ${ }^{1}$ Wushuang Cao, ${ }^{1}$ Shuo Yang, ${ }^{7}$ Fen-Ting Liu, ${ }^{7}$ Lifeng Tian, ${ }^{3}$ Han Zeng, ${ }^{3}$ Huiling Xu, ${ }^{4}$ Shumin Qiu, ${ }^{4}$ Lihua Yang, ${ }^{5}$ Xiao Chen, ${ }^{5}$ Xiaoqin Pan, ${ }^{6}$ Xiaoyun Wu, ${ }^{6}$ Ben W Mol (i) , ${ }^{2}$ Rong Li, ${ }^{7}$ Dan Zhang (i) ${ }^{1}$

To cite: Hu K-L, Gan K, Wang R, et al. Vitamin D supplementation prior to in vitro fertilisation in women with polycystic ovary syndrome: a protocol of a multicentre randomised, doubleblind, placebo-controlled clinical trial. BMJ Open 2020;10:e041409. doi:10.1136/ bmjopen-2020-041409

- Prepublication history for this paper is available online. To view these files, please visit the journal online (http://dx.doi. org/10.1136/bmjopen-2020041409).

Received 08 June 2020 Revised 04 November 2020 Accepted 09 November 2020

Check for updates

(C) Author(s) (or their employer(s)) 2020. Re-use permitted under CC BY-NC. No commercial re-use. See rights and permissions. Published by BMJ.

For numbered affiliations see end of article.

Correspondence to

Dr Dan Zhang;

zhangdan@zju.edu.cn and

Dr Rong Li;

roseli001@sina.com

\section{ABSTRACT}

Introduction Polycystic ovary syndrome (PCOS) is one of the leading causes of female infertility, affecting around $5 \%$ of women of childbearing age in China. Vitamin D insufficiency is common in women with PCOS and is associated with lower live birth rates. However, evidence regarding the effectiveness of vitamin $D$ supplementation in women with PCOS is inconclusive. This multicentre randomised, double-blinded, placebo-controlled trial aims to evaluate the effectiveness of vitamin $D$ supplementation prior to in vitro fertilisation (IVF) on the live birth rate in women with PCOS.

Methods and analysis We plan to enrol women with PCOS scheduled for IVF. After informed consent, eligible participants will be randomised in a 1:1 ratio to receive oral capsules of $4000 \mathrm{IU}$ vitamin D per day or placebo for around 12 weeks until the day of triggering. All IVF procedures will be carried out routinely in each centre. The primary outcome is live birth after the first embryo transfer. The primary analysis will be by intention-to-treat analysis. To demonstrate or refute that treatment with vitamin D results in a 10\% higher live birth rate than treatment with placebo, we need to recruit 860 women (48\% vs $38 \%$ difference, anticipating $10 \%$ loss to follow-up and noncompliance, significance level 0.05 and power $80 \%$ ). Ethics and dissemination This study has been approved by the Ethics Committee in Women's Hospital of Zhejiang University on 2 March 2020 (reference number: IRB20200035-R). All participants will provide written informed consent before randomisation. The results of the study will be submitted to scientific conferences and a peerreviewed journal.

Trial registration number NCT04082650.

\section{INTRODUCTION}

Polycystic ovary syndrome (PCOS) is a heterogeneous clinical condition characterised by androgen excess, oligo-ovulation
Strengths and limitations of this study

This is a multicentre randomised, double-blinded, placebo-controlled trial.

- The intended sample size is large with 860 participants.

- Despite the large sample size, our study is not designed to detect a small difference.

or anovulation, and polycystic ovary morphology. ${ }^{1}$ PCOS is one of the common causes of infertility and it affects around $5.6 \%$ of women with childbearing age in China. ${ }^{2}$ In women with PCOS, the proportion of severe vitamin $\mathrm{D}$ deficiency (less than $10 \mathrm{ng} / \mathrm{mL}$ ) was significantly higher than that in women without PCOS (44\% vs $11 \%)$. $^{3}$

The most reliable determinant of serum vitamin $\mathrm{D}$ is the serum concentration of 25-hydroxyvitamin D (25-OHD), with a half-life of $2-3$ weeks. ${ }^{45}$ Serum vitamin D levels show seasonal variations, with the highest levels in autumn and the lowest levels in spring. ${ }^{6}$ The optimal concentration of serum $25-\mathrm{OHD}$ is $30 \mathrm{ng} / \mathrm{mL}$ or above, whereas concentrations below $20 \mathrm{ng} / \mathrm{mL}$ $(50 \mathrm{nmol} / \mathrm{L})$ and $30 \mathrm{ng} / \mathrm{mL}(75 \mathrm{nmol} / \mathrm{L})$ are considered deficiency and insufficiency, respectively. In China, around $88 \%$ of individuals aged over 15 years have vitamin D deficiency or insufficiency. ${ }^{7} 8$ PCOS women with vitamin D deficiency are less likely to ovulate and have a lower live birth rate, whereas in women with unexplained infertility, vitamin D deficiency 


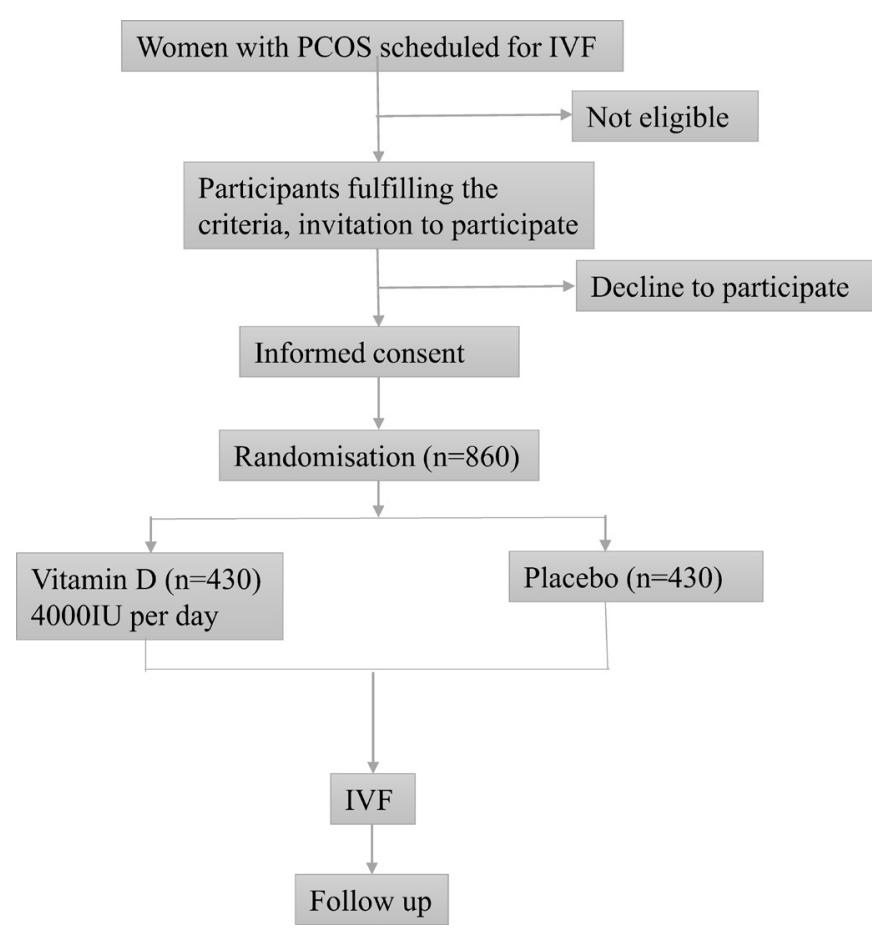

Figure 1 Flowchart of recruitment. IVF, in vitro fertilisation; PCOS, polycystic ovary syndrome.

does not significantly reduce the live birth rate. ${ }^{9}$

Recent studies have shown that vitamin D supplementation improves insulin resistance and lipid metabolism, reduces testosterone levels, inflammatory indicators and serum anti-mullerian hormone (AMH) levels, and regulates the menstrual cycle in women with PCOS. ${ }^{10-21}$ In contrast, these effects are not significant in non-PCOS women with vitamin D insufficiency ${ }^{10-21}$ A recent study indicates that serum vitamin D levels are associated positively with fertilisation rate but not clinical pregnancy and live birth rates following in vitro fertilisation (IVF) ${ }^{22}$ These findings indicate that vitamin $\mathrm{D}$ treatment functions mainly in women with PCOS.

The role of vitamin D supplementation in IVF is inconclusive. Vitamin D deficiency does not significantly impair pregnancy rates among infertile women undergoing frozen-thawed embryo transfer cycles. ${ }^{23}{ }^{24}$ A trial showed that a single dose of 300000 IU vitamin D supplementation 2 months prior to ovarian stimulation significantly increases the endometrium thickness in PCOS women who undergo intrauterine insemination but does not significantly improve ongoing pregnancy rate $(38 \%$ vs $33 \%) .{ }^{25}$ However, another trial showed that concurrent use of vitamins $\mathrm{D}$ and $\mathrm{E}$ for 2 months prior to embryo transfer significantly increased the clinical pregnancy rate from $23 \%$ to $62 \%$ and live birth rate from $16 \%$ to $43 \%$ in women with PCOS undergoing IVF. ${ }^{26}$ Due to the inconsistent evidence, no consensus has been reached regarding whether vitamin $\mathrm{D}$ should be routinely used in women with PCOS before IVF. In China, the current recommended upper limit for vitamin D supplements in reproductive women is $2000 \mathrm{IU} /$ day. ${ }^{27}$ However, this dose recommendation is conservative as $60 \%$ of Chinese adults with vitamin D deficiency taking $2000 \mathrm{IU} /$ day for 3 months fail to reach the optimal 25 -OHD levels $(\geq 75 \mathrm{nmol} / \mathrm{L}) .{ }^{27} 28$ Therefore, we plan a multicentre randomised, doubleblind, placebo-controlled trial to evaluate whether the treatment of vitamin D at a dose of $4000 \mathrm{IU}$ for around 12 weeks prior to IVF improves live birth rates in women with PCOS.

\section{MATERIALS AND METHODS Design, setting and participants}

We plan a multicentre randomised, double-blind, placebo-controlled trial. The participating centres include Women's Hospital of Zhejiang University (Hangzhou), Peking University Third Hospital (Beijing), Jiangxi Maternal and Child Health Hospital (Nanchang), Fujian Maternity and Child Health Hospital (Fuzhou), Jinhua People's Hospital (Jinhua) and Huzhou Maternity and Child Health Care Hospital (Huzhou).

The detailed inclusion and exclusion criteria for participants are presented below.

Inclusion criteria:

1. Women aged 20-42 years.

2. Diagnosed with PCOS (Rotterdam criteria). ${ }^{1}$

3. Scheduled for IVF.

4. Written informed consent.

Exclusion criteria:

1. Women who had three or more failed IVF cycles.

2. Women scheduled for preimplantation genetic testing.

3. Known vitamin D allergy.

4. Women with a history of chronic absorption syndrome or bile dysplasia, or parathyroid dysfunction, or kidney stones, or blood calcium ion concentration greater than $2.6 \mathrm{mmol} / \mathrm{L}$ (normal value: $2.25-2.75 \mathrm{mmol} / \mathrm{L}$ (9$11 \mathrm{mg} / \mathrm{dL})$, or hyperphosphataemia $(1.61 \mathrm{mmol} / \mathrm{L})$, metabolic-related bone disease, or chronic diseases that may cause bone abnormalities (liver and kidney insufficiency).

5. Women receiving treatments for tuberculosis, convulsions and epilepsy because medications treating these diseases may affect the metabolism of vitamin $\mathrm{D}$.

6. Women undergoing an IVF treatment with donor oocytes.

\section{Recruitment and randomisation}

PCOS women scheduled for IVF will be invited to participate in this study if they fulfil the eligibility criteria. Researchers in each centre will explain the study protocol to potential participants. After signing informed consent, participants will be enrolled and randomised to the vitamin D group or the placebo group (figure 1). We will use a computer-generated randomisation list in a 1:1 ratio, with a variable block size of four or six stratified by study centre. The randomisation list will be prepared by an independent statistician who will not participate in the recruitment and the list will not be available to 
investigators in any centre. The randomisation list will be implemented with the use of sequentially numbered identical study-drug containers. The containers will be labelled centrally by two researchers who will not be involved in the participants' recruitment.

\section{Blinding}

Only the two researchers who labeled the containers will be aware of the allocation. Participants and other researchers will be unaware of the treatment allocation. Vitamin D capsules are produced by a licensed pharmaceutical company (Sinopharm Xingsha Pharmaceuticals (Xiamen) Co). Placebo capsules, with identical package, size, colour and appearance to Vitamin D capsules, are produced by the same manufacturer. In general, there should be no need to unblind the allocation. If urgent unbinding to participants is necessary, the allocation will be disclosed to the treating physician.

\section{Intervention and comparator \\ The vitamin $\mathrm{D}$ group}

Participants in the intervention group will be treated with vitamin D $4000 \mathrm{IU} /$ day (800 IU per capsule, take five capsules once each day) for around 12 weeks (begins from $6-10$ weeks prior to the ovarian stimulation till the triggering day).

\section{The placebo group}

Participants in the placebo group will be treated with five placebo capsules per day for the same duration.

\section{Outcomes}

\section{Primary outcome}

The primary outcome is live birth after the first embryo transfer. Live birth is defined as the delivery of at least one baby after 24 weeks of gestation that exhibits any sign of life. Pregnancies that occurs before the first embryo transfer but after randomisation will be included.

\section{Secondary outcomes}

Pregnancies after the first embryo transfer will not be considered in the following pregnancy-related secondary outcomes except for cumulative live birth.

- Cumulative live birth: defined as live birth resulting from pregnancies that occur within 6 months after randomisation.

- Clinical pregnancy: defined as at least one gestational sac on ultrasound at around 7 weeks gestation with the detection of heart-beat activity.

- Ongoing pregnancy: defined as pregnancy with detectable heart rate at 12 weeks gestation or beyond.

- Biochemical pregnancy: defined as a positive pregnancy test.

- Ectopic pregnancy: defined as embryo implanted at any site other than the endometrial lining of the uterus cavity.

- Miscarriage: defined as a positive pregnancy test but no detectable heart rate before 24 weeks gestation.

- Number of available embryos for transfer;
- Moderate or severe ovarian hyperstimulation syndrome (OHSS): defined according to the Golan's criteria. ${ }^{29}$ Moderate OHSS is diagnosed by the presence of ascites on ultrasonography in addition to abdominal distension and discomfort with or without nausea, vomiting and/or diarrhoea. Severe OHSS is diagnosed when there is clinical evidence of ascites and/or hydrothorax or breathing difficulties with or without haemoconcentration, coagulation abnormalities and diminished renal function.

In case of pregnancy, we will report the following endpoints:

- Pre-eclampsia: defined as the development of gestational hypertension with proteinuria $(\geq 300 \mathrm{mg} / 24$ hour urine collection or $30 \mathrm{mg} / \mathrm{dL}$ in single urine sample) after 20 weeks of gestation.

- Gestational hypertension: defined as the development of blood pressure greater than $140 / 90 \mathrm{~mm} \mathrm{Hg}$ after pregnancy without proteinuria or other signs of pre-eclampsia.

- Gestational diabetes mellitus: defined as carbohydrate intolerance of variable severity with onset or first recognition during pregnancy as determined from the diagnosis in the obstetrical medical record.

- Premature rupture of membrane (PROM) rate: defined as rupture of the amniotic membranes before the onset of labour, including PROM at term and preterm PROM.

- Preterm delivery: defined as delivery of a fetus at less than 37 and more than 24weeks' gestational age (classified as spontaneous or iatrogenic);

- Placenta previa: defined as a placenta that is implanted over or very close to the internal cervical orifice.

- Postpartum haemorrhage: defined as the loss of $500 \mathrm{~mL}$ of blood or more after completion of the third stage of labour.

- Birth weight.

- 1-minute and 5-minute Apgar score.

- 5-minute Apgar score $<7$.

- Stillbirth: defined as the absence of signs of life at or after birth.

- Serum 25-OHD levels at the triggering day and the pregnancy test day.

- Vitamin D binding protein, insulin and calcium ion concentration at the triggering day.

\section{Standard IVF procedure}

The oral contraceptive capsule will be used for participants with an irregular menstrual cycle (longer than 35 days or shorter than 21 days) at least one cycle prior to ovarian stimulation. The long or short GonadotrophinReleasing Hormone (GnRH) agonist or GnRH antagonist protocol will be used. In the long GnRH agonist protocol, GnRH agonist will be administered in the midluteal phase of the cycle preceding ovarian stimulation. In the short GnRH agonist protocol, GnRH agonist will be administered on day 2 of the ovarian stimulation cycle. In the GnRH antagonist protocol, GnRH antagonist will 


\begin{tabular}{|c|c|c|c|c|c|c|c|c|c|c|c|c|c|}
\hline \multirow[t]{2}{*}{ Evaluation } & \multirow[t]{2}{*}{ Enrollment } & \multirow[t]{2}{*}{ Allocation } & \multirow{2}{*}{$\begin{array}{l}\text { Online } \\
\text { supervision }\end{array}$} & \multirow{2}{*}{$\begin{array}{l}\text { Oocyte } \\
\text { collecting } \\
\text { day }\end{array}$} & \multicolumn{2}{|c|}{ Embryo transfer } & \multirow{2}{*}{$\begin{array}{l}\text { Pregnancy } \\
\text { test day }\end{array}$} & \multirow{2}{*}{$\begin{array}{l}35 \text { days } \\
\text { after ET }\end{array}$} & \multirow{2}{*}{$\begin{array}{l}12 \text { weeks } \\
\text { gestation }\end{array}$} & \multirow{2}{*}{$\begin{array}{l}24 \text { weeks } \\
\text { gestation }\end{array}$} & \multirow{2}{*}{$\begin{array}{l}37 \text { weeks } \\
\text { gestation }\end{array}$} & \multirow{2}{*}{$\begin{array}{l}\text { Delivery } \\
\text { day }\end{array}$} & \multirow{2}{*}{$\begin{array}{l}\text { Six weeks } \\
\text { after delivery }\end{array}$} \\
\hline & & & & & Fresh ET & FET & & & & & & & \\
\hline Informed consent & & $\boldsymbol{\Delta}$ & & & & & & & & & & & \\
\hline Medical/treatment history & $\boldsymbol{\Delta}$ & & & & & & & & & & & & \\
\hline Physical exam & $\boldsymbol{\Delta}$ & & & $\boldsymbol{\Delta}$ & & & & & & & & & \\
\hline Ultrasonography & $\boldsymbol{\Delta}$ & & & $\boldsymbol{\Delta}$ & & & & $\boldsymbol{\Delta}$ & $\boldsymbol{\Delta}$ & & & & \\
\hline Steroid hormone & & $\boldsymbol{\Delta}$ & & $\boldsymbol{\Delta}$ & & & & & & & & & \\
\hline $\begin{array}{l}\text { Liver/renal function, metabolic } \\
\text { test }\end{array}$ & & $\boldsymbol{\Delta}$ & & & & & & & & & & & \\
\hline SEQ within 3 months & & & & $\boldsymbol{\Delta}$ & & & & & & & & & \\
\hline FFQ within 3 months & & & & $\boldsymbol{\Delta}$ & & & & & & & & & \\
\hline Allocation & & $\boldsymbol{\Delta}$ & & & & & & & & & & & \\
\hline IVF-ET protocol & & & & & $\boldsymbol{\Delta}$ & $\boldsymbol{\Delta}$ & & & & & & & \\
\hline Pregnancy test & & & & & & & $\boldsymbol{\Delta}$ & & & & & & \\
\hline $\begin{array}{l}\text { Record of pregnancy/obstetric } \\
\text { complications }\end{array}$ & & & & & & & & $\boldsymbol{\Delta}$ & $\boldsymbol{\Delta}$ & $\boldsymbol{\Delta}$ & $\boldsymbol{\Delta}$ & & \\
\hline $\begin{array}{l}\text { Record of maternal and infant } \\
\text { health after delivery }\end{array}$ & & & & & & & & & & & & $\boldsymbol{\Delta}$ & $\boldsymbol{\Delta}$ \\
\hline Serum sample of female & $\boldsymbol{\Delta}$ & & & $\boldsymbol{\Delta}$ & & & $\boldsymbol{\Delta}$ & & & & & & \\
\hline Drug recycling and reporting & & & & $\boldsymbol{\Delta}$ & & & & & & & & & \\
\hline Report form of OHSS & & & & & $\boldsymbol{\Delta}$ & $\boldsymbol{\Delta}$ & $\boldsymbol{\Delta}$ & & & & & & \\
\hline $\begin{array}{l}\text { Report form of concomitant } \\
\text { drug use }\end{array}$ & & $\boldsymbol{\Delta}$ & $\boldsymbol{\Delta}$ & $\boldsymbol{\Delta}$ & $\boldsymbol{\Delta}$ & $\boldsymbol{\Delta}$ & $\boldsymbol{\Delta}$ & $\boldsymbol{\Delta}$ & & & & & \\
\hline Report form of adverse event & & & $\boldsymbol{\Delta}$ & $\boldsymbol{\Delta}$ & $\boldsymbol{\Delta}$ & $\boldsymbol{\Delta}$ & $\boldsymbol{\Delta}$ & $\boldsymbol{\Delta}$ & & & & & \\
\hline Phone call follow up & & & & & & & & & $\boldsymbol{\Delta}$ & $\boldsymbol{\Delta}$ & $\boldsymbol{\Delta}$ & $\boldsymbol{\Delta}$ & $\boldsymbol{\Delta}$ \\
\hline Medical record review & & & & & & & & & & & & $\Delta$ & \\
\hline
\end{tabular}

Figure 2 Study visits. ET, embryo transfer; FFQ, food frequency questionnaire; IVF, in vitro fertilisation; OHSS, ovarian hyperstimulation syndrome; SEQ, sunshine exposure questionnaire.

be administered according to the follicle development and serum estradiol levels (around 5 days or 6 days after ovarian stimulation). The starting dose of gonadotrophins will be $75-225 \mathrm{IU}$. The dose of gonadotrophins will be adjusted according to the ovarian response evaluated by follicle development and serum estradiol levels. HCG 4000-8000 IU will be used to trigger ovulation when at least 3 follicles with a diameter of at least $18 \mathrm{~mm}$ are present, and oocyte retrieval will be carried out 36-37 hours after triggering.

\section{Fertilisation, embryo transfer and luteal support}

IVF will be performed 4-6hours after oocyte retrieval by using either conventional IVF or intracytoplasmic sperm injection. Embryo transfer strategies will be used according to local protocol. Up to two embryos will be transferred at the cleavage or blastocyst stage. Endometrium thickness will be measured by transvaginal ultrasound 1 day prior to luteal support. Luteal support begins from the beginning of ovulation day. Embryo transfer will be performed on day 3 , day 5 or day 6 after ovulation.

\section{Research visits}

Detailed study visits are presented in figure 2. After informed consent, baseline data, including 25-OHD levels, will be collected. We will monitor and promote compliance by encouraging participants to record the daily intake of the dosage of study medication during the trial through an online tool. Meanwhile, trial coordinators will contact participants regularly to monitor compliance.

\section{Vitamin D measurement}

Serum vitamin D levels will be measured before the intervention, on the triggering day and at the pregnancy test day for all participants. We also plan to measure vitamin D levels in each trimester for participants who will get delivery in two centres, Peking University Third Hospital and Women's Hospital of Zhejiang University. These data will be used to generate secondary hypotheses.

\section{Sample size calculation}

The current live birth rate of women with PCOS after the first embryo transfer per started cycle is $38 \%$ in Women's Hospital of Zhejiang University. To demonstrate an increase of $10 \%$ in the live birth rate in the vitamin D treatment group (48\%), a total of 768 participants are required (power $80 \%$ and $\alpha$ error: $5 \%$ ). Taking into account possible protocol violation and loss of follow-up of around $10 \%$, we plan to enrol 860 participants (430 per arm).

\section{Statistical methods}

Statistical analysis will be conducted according to the intention-to-treat principle. We will present descriptive statistics for baseline variables. For continuous characteristics, distribution between the two groups will be presented with means and SDs or medians and IQRs according to the normality of data. For categorical characteristics, we will present the proportions of the two arms. The incidence of binary outcomes will be estimated for each treatment group and the differences between groups will be presented using $\chi^{2}$ test and risk ratios and 
their $95 \%$ CIs. For continuous outcomes, means and SDs or medians and IQRs will be presented for each treatment group and compared using the Student's t-test or the Mann-Whitney test. Finally, a per-protocol analysis will be conducted but these results would be considered exploratory only. Details of the analysis will be described in a separate statistical analysis plan which will be signed off prior to data-lock.

\section{Study duration}

The trial is expected to start in July 2020 with a duration of 18 months for recruitment. It is expected to recruit the last participant in December 2021.

\section{Patient and public involvement}

Patients and the public were not involved in the development of the research question or study design. They will not be involved in the recruitment, conduct, or report of the study. The study results will be disseminated to study participants by social media.

\section{Data monitoring committee and interim analysis}

An independent Data Safety Monitoring Committee (DSMC) formed by at least two clinicians and one statistician who are independent of the sponsor without competing interests will monitor the conduct of the trial. An interim analysis will be performed 6 months after the recruitment of the first 300 participants. The DSMC will be asked to assess ongoing pregnancy, as the primary endpoint live birth would not be achieved in most participants at the interim analysis. The study could be stopped prematurely based on the advice of the DSMC.

\section{Adverse events monitoring}

Adverse events will be closely monitored during this study. If a participant shows suspected drug-related severe discomfort symptoms, abnormal signs, abnormal liver and kidney function or abnormal blood routine results, then she will be instructed to discontinue the trial medication. Researchers will continue to observe the symptoms and signs until they disappear or the test results are normal. A previous randomised controlled trial (RCT) showed that adults taking vitamin D $4000 \mathrm{IU} /$ day for 3 months with serum vitamin $\mathrm{D}$ concentrations reaching $81.3-115.3 \mathrm{nmol} / \mathrm{L}$ did not present safety concerns. ${ }^{30}$ Common adverse events related to vitamin D supplements are mainly bone and joint pain, swelling, itching of the skin, vomiting, constipation or diarrhoea, nausea, etc. Rare adverse events include allergic reactions. A severe adverse event is defined as an event that occurs during the study and meets one of the following criteria: death, life threatening, causing severe or permanent disability, congenital malformation or birth defect, and prolonged hospitalisation. No severe adverse event (SAE) in relation to vitamin $\mathrm{D}$ has been reported in the literature.

\section{Ethics and dissemination}

This study has been approved by the Ethics Committee in Women's Hospital of Zhejiang University on 2 March 2020 (reference number: IRB-20200035-R). Protocol modifications after the recruitment of the first participant will not be allowed unless it is necessary and additional ethical approval will be required. All participants must provide written informed consent before participating in the trial. Data handling will be done anonymously, with only the participant code available in the central database. All personal information on potential and enrolled participants out of the scope of this trial will not be collected, shared or maintained to protect confidentiality before, during and after the trial. The results of the study will be submitted to scientific conferences in reproductive medicine and a peer-reviewed journal.

\section{Author affiliations}

${ }^{1}$ Department of Reproductive Endocrinology, Key Laboratory of Reproductive Genetics, Ministry of Education, Women's Hospital, School of Medicine, Zhejiang University, Hangzhou, China

${ }^{2}$ Department of Obstetrics and Gynaecology, Monash University, Clayton, Victoria, Australia

${ }^{3}$ Reproductive Medicine Center, Jiangxi Maternal and Child Health Hospital, Nanchang, China

${ }^{4}$ Reproductive Medicine Center, Fujian Maternity and Child Health Hospital, Affiliated Hospital of Fujian Medical University, Fuzhou, China

${ }^{5}$ Department of Reproductive Medicine, Jinhua People's Hospital, Jinhua, China ${ }^{6}$ Department of Obstetrics and Gynaecology, Huzhou Maternity and Child Health Care Hospital, Huzhou, China

${ }^{7}$ Center for Reproductive Medicine, Department of Obstetrics and Gynaecology, Peking University Third Hospital, Beijing, China

Acknowledgements We thank Dr Jianyu Qu for providing inputs on the trial management. We also thank all participants who will be involved in this trial.

Contributors Study concept and design: K-LH, RW, WL, BWM, GK, RL and DZ. Drafting of the manuscript: K-LH, RW, WL and BWM. Critical revision of the manuscript for important intellectual content: RL and DZ. Study supervision: BWM, RL and DZ. Recruitment: K-LH, GK, SY, F-TL, HZ, HX, SQ, LY, XC, XP and XW. Statistical analysis: RW and WL. Critical discussion: K-LH, GK, YL, YW, RC, WC, SY, F-TL, LT, HZ, HX, SQ, LY, XC, XP, XW, QW, BZ, LZ, SZ, RL and DZ.

Funding This study is supported by the National Key Research and Development Program of China (Nos 2018YFC1005003 and 2017YFC1001003), the National Natural Science Foundation of China (Nos 81974224 and 81771535), the Fundamental Research Funds for the Central Universities, the Natural Science Foundation of Zhejiang Province (No. LZ18H040001), Zhejiang University

Scholarship for Outstanding Doctoral Candidates, and Zhejiang University Education Foundation Global Partnership Fund.

Competing interests This trial is partly funded by Sinopharm Xingsha Pharmaceuticals (Xiamen) Co Ltd, which is the manufacturer of vitamin D. The funder has no role in the trial design, trial conduct, data collection, data analysis or manuscript preparation. All authors report no conflict of interest.

Patient and public involvement Patients and/or the public were not involved in the design, or conduct, or reporting, or dissemination plans of this research.

Patient consent for publication Not required.

Provenance and peer review Not commissioned; externally peer reviewed.

Open access This is an open access article distributed in accordance with the Creative Commons Attribution Non Commercial (CC BY-NC 4.0) license, which permits others to distribute, remix, adapt, build upon this work noncommercially, and license their derivative works on different terms, provided the original work is properly cited, appropriate credit is given, any changes made indicated, and the use is non-commercial. See: http://creativecommons.org/ licenses/by-nc/4.0/.

\section{ORCID iDs}

Rui Wang http://orcid.org/0000-0002-6622-8134

Ben W Mol http://orcid.org/0000-0001-8337-550X

Dan Zhang http://orcid.org/0000-0003-1295-4795 


\section{REFERENCES}

1 Group REA-SPCW. Revised 2003 consensus on diagnostic criteria and long-term health risks related to polycystic ovary syndrome. Fertil Steril 2004;81:19-25.

2 Li R, Zhang Q, Yang D, et al. Prevalence of polycystic ovary syndrome in women in China: a large community-based study. Hum Reprod 2013;28:2562-9.

3 Li HWR, Brereton RE, Anderson RA, et al. Vitamin D deficiency is common and associated with metabolic risk factors in patients with polycystic ovary syndrome. Metabolism 2011;60:1475-81.

4 Pearce SHS, Cheetham TD. Diagnosis and management of vitamin D deficiency. BMJ 2010;340:b5664.

5 Holick MF, Binkley NC, Bischoff-Ferrari HA, et al. Evaluation, treatment, and prevention of vitamin $D$ deficiency: an endocrine Society clinical practice guideline. J Clin Endocrinol Metab 2011;96:1911-30.

6 Niculescu DA, Capatina CAM, Dusceac R, et al. Seasonal variation of serum vitamin D levels in Romania. Arch Osteoporos 2017;12:113.

7 Li M, Lv F, Zhang Z, et al. Establishment of a normal reference value of parathyroid hormone in a large healthy Chinese population and evaluation of its relation to bone turnover and bone mineral density. Osteoporos Int 2016;27:1907-16.

8 Lu H-K, Zhang Z, Ke Y-H, HK L, YH K, et al. High prevalence of vitamin D insufficiency in China: relationship with the levels of parathyroid hormone and markers of bone turnover. PLoS One 2012;7:e47264.

9 Butts SF, Seifer DB, Koelper N, et al. Vitamin D deficiency is associated with poor ovarian stimulation outcome in PCOS but not unexplained infertility. J Clin Endocrinol Metab 2019;104:369-78.

10 Thys-Jacobs S, Donovan D, Papadopoulos A, et al. Vitamin D and calcium dysregulation in the polycystic ovarian syndrome. Steroids 1999;64:430-5.

11 Dastorani M, Aghadavod E, Mirhosseini N, et al. The effects of vitamin D supplementation on metabolic profiles and gene expression of insulin and lipid metabolism in infertile polycystic ovary syndrome candidates for in vitro fertilization. Reproductive Biology and Endocrinology 2018;16:94.

12 Ostadmohammadi V, Jamilian M, Bahmani F, et al. Vitamin D and probiotic co-supplementation affects mental health, hormonal, inflammatory and oxidative stress parameters in women with polycystic ovary syndrome. J Ovarian Res 2019;12:5.

13 Seyyed Abootorabi M, Ayremlou P, Behroozi-Lak T, et al. The effect of vitamin $D$ supplementation on insulin resistance, visceral fat and adiponectin in vitamin D deficient women with polycystic ovary syndrome: a randomized placebo-controlled trial. Gynecol Endocrinol 2018;34:489-94.

14 Nasri K, Akrami S, Rahimi M, et al. The effects of vitamin D and evening primrose oil co-supplementation on lipid profiles and biomarkers of oxidative stress in vitamin D-deficient women with polycystic ovary syndrome: a randomized, double-blind, placebocontrolled trial. Endocr Res 2018;43:1-10.

15 Pal L, Berry A, Coraluzzi L, et al. Therapeutic implications of vitamin $\mathrm{D}$ and calcium in overweight women with polycystic ovary syndrome. Gynecol Endocrinol 2012;28:965-8.

16 Asemi Z, Foroozanfard F, Hashemi T, et al. Calcium plus vitamin $\mathrm{D}$ supplementation affects glucose metabolism and lipid concentrations in overweight and obese vitamin $D$ deficient women with polycystic ovary syndrome. Clin Nutr 2015;34:586-92.

17 Jamilian M, Foroozanfard F, Rahmani E, et al. Effect of two different doses of vitamin D supplementation on metabolic profiles of insulinresistant patients with polycystic ovary syndrome. Nutrients 2017;9. doi:10.3390/nu9121280. [Epub ahead of print: 2411 2017].

18 Foroozanfard F, Talebi M, Samimi M, et al. Effect of two different doses of vitamin D supplementation on metabolic profiles of insulin-resistant patients with polycystic ovary syndrome: a randomized, double-blind, placebo-controlled trial. Horm Metab Res 2017;49:612-7.

19 Maktabi M, Chamani M, Asemi Z. The effects of vitamin D supplementation on metabolic status of patients with polycystic ovary syndrome: a randomized, double-blind, placebo-controlled trial. Horm Metab Res 2017;49:493-8.

20 Karadağ C, Yoldemir T, Yavuz DG. Effects of vitamin D supplementation on insulin sensitivity and androgen levels in vitaminD-deficient polycystic ovary syndrome patients. J Obstet Gynaecol Res 2018;44:270-7.

21 Javed Z, Papageorgiou M, Deshmukh $\mathrm{H}$, et al. A randomized, controlled trial of vitamin D supplementation on cardiovascular risk factors, hormones, and liver markers in women with polycystic ovary syndrome. Nutrients 2019;11. doi:10.3390/nu11010188. [Epub ahead of print: 17 Jan 2019].

22 Liu X, Zhang W, Xu Y, et al. Effect of vitamin D status on normal fertilization rate following in vitro fertilization. Reprod Biol Endocrinol 2019;17:59

23 van de Vijver A, Drakopoulos P, Van Landuyt L, et al. Vitamin D deficiency and pregnancy rates following frozen-thawed embryo transfer: a prospective cohort study. Hum Reprod 2016;31:1749-54.

24 Aflatoonian A, Arabjahvani F, Eftekhar M, et al. Effect of vitamin D insufficiency treatment on fertility outcomes in frozen-thawed embryo transfer cycles: a randomized clinical trial. Iran J Reprod Med 2014;12:595-600.

25 Asadi M, Matin N, Frootan M, et al. Vitamin D improves endometrial thickness in PCOS women who need intrauterine insemination: a randomized double-blind placebo-controlled trial. Arch Gynecol Obstet 2014;289:865-70.

26 Fatemi F, Mohammadzadeh A, Sadeghi MR, et al. Role of vitamin $E$ and $D_{3}$ supplementation in Intra-Cytoplasmic sperm Injection outcomes of women with polycystic ovarian syndrome: a double blinded randomized placebo-controlled trial. Clin Nutr ESPEN 2017;18:23-30.

27 Yao P, Lu L, Hu Y, et al. A dose-response study of vitamin D3 supplementation in healthy Chinese: a 5-arm randomized, placebocontrolled trial. Eur J Nutr 2016;55:383-92.

28 Yao P, Sun L, Lu L, et al. Effects of Genetic and Nongenetic Factors on Total and Bioavailable 25(OH)D Responses to Vitamin D Supplementation. J Clin Endocrinol Metab 2017;102:100-10.

29 Herman A, Ron-El R, Golan A, et al. Overstimulated cycles under low-dose gonadotrophins in patients with polycystic ovary syndrome: characterization and management. Hum Reprod 1993;8:30-4.

30 Burt LA, Billington EO, Rose MS, et al. Effect of high-dose vitamin D supplementation on volumetric bone density and bone strength: a randomized clinical trial. JAMA 2019;322:736-45. 